\title{
Identification of key genes and pathways in uterine leiomyosarcoma through bioinformatics analysis
}

\author{
YUQIN ZANG, LINA GU, YANFANG ZHANG, YINGMEI WANG and FENGXIA XUE \\ Department of Gynecology and Obstetrics, Tianjin Medical University General Hospital, Tianjin 300052, P.R. China
}

Received October 29, 2017; Accepted March 20, 2018

DOI: $10.3892 / \mathrm{ol} .2018 .8503$

\begin{abstract}
Uterine leiomyosarcoma (uLMS) is a rare but malignant gynaecological tumour with a poor survival outcome. The present study was aimed at identifying the key genes and pathways in the development of uLMS through bioinformatics analysis. To minimize the frequency of false-positive results of the bioinformatics analysis, 3 microarrays including GSE764, GSE64763 and GSE68312 were downloaded from Gene Expression Omnibus (GEO). Differentially expressed genes (DEGs) were screened out using the online tool GEO2R. Then, Gene Ontology and Kyoto Encyclopaedia of Genes and Genomes pathway enrichment analyses were performed using the Database for Annotation, Visualization and Integrated Discovery. Finally, a protein-protein interaction (PPI) network of the DEGs was constructed using Cytoscape, and module analysis was conducted using the plug-in MCODE. A total of 95 DEGs including 21 upregulated genes and 74 downregulated genes were identified. The upregulated DEGs were annotated with 'DNA metabolic process', 'nucleobase-containing compound biosynthetic process' and 'cellular macromolecule biosynthetic process', while the downregulated DEGs were annotated with 'cellular response to chemical stimulus', 'movement of cell or subcellular component' and 'response to inorganic substances'. The results of the PPI network analysis demonstrated that matrix metallopeptidase 9, apolipoprotein E, cyclin E1 and syndecan 1 were the predominant upregulated genes in uLMS. Additionally, the genes in the main module were enriched in 'proteoglycans in cancer', 'p53 signalling pathway' and 'extracellular matrix-receptor interaction'. The key genes and pathways identified in the present study may provide valuable clues for clarifying the molecular mechanism
\end{abstract}

Correspondence to: Dr Yingmei Wang or Dr Fengxia Xue, Department of Gynecology and Obstetrics, Tianjin Medical University General Hospital, 154 Anshan Road, He Ping, Tianjin 300052, P.R. China

E-mail: wangyingmei1978@126.com

E-mail: fengxiaxue1962@gmail.com

Key words: uterine leiomyosarcoma, bioinformatic analysis, microarray, differentially expressed genes, functional enrichment analysis, protein-protein interaction underlying the development of uLMS and demonstrate promise for use as diagnostic markers and therapeutic targets.

\section{Introduction}

Uterine sarcoma is a rare but aggressive malignant gynecological tumor with unknown aetiology and pathogenesis. Uterine leiomyosarcoma (uLMS) is the most common histological subtype of uterine sarcoma originating in the smooth muscles of the myometrium. It accounts for only $1 \%$ of all uterine malignancies; however, it contributes to a considerable proportion of uterine cancer deaths (1). With poor biological characteristics, the overall 5-year survival rate for uLMS is only $25 \%$ (2). Surgical treatment is the mainstay of therapy for uLMS; however, 50-71\% of these patients would develop recurrence (1). Adjuvant radiotherapy and chemotherapy have minimal effect on improving patient survival $(3,4)$. Hormone therapy appears to be effective for hormone receptor-positive uLMS; however, the evidence for this is inadequate (4). Targeted therapy has been developed rapidly in recent years and it is expected to be a promising treatment for uLMS. Thus, it is necessary to explore the molecular aetiology and pathogenesis of uLMS and to search for therapeutic molecular targets.

The study of uLMS is challenging because of its rarity. Recently, the genome-wide DNA microarray, which is a high-throughput platform for the analysis of gene expression, has been regarded as an efficient tool for detecting molecular changes in diseases. There are a few microarrays of uLMS in the Gene Expression Omnibus (GEO) repository; however, no detailed bioinformatics analysis has been performed. The most relevant study was conducted by Barlin et al (5). They examined and compared the expression profiles of uLMS cells, fibroids and normal myometrium for the identification of molecular subtypes and correlation with clinical outcomes. In addition, they found that some genes that are related to cell-cycle regulation, such as CDC7, CDC20, GTSE1, CCNA2, $C C N B 1$ and $C C N B 2$ were overexpressed in uLMS. However, the interactions among the differentially expressed genes (DEGs) still remain poorly understood.

Consequently, we performed bioinformatic analysis to explore the changes in the expression of mRNA and interactions among the DEGs during the occurrence of uLMS. To minimize the frequency of false-positive results of the microarray analysis, we used 3 microarrays including GSE764, 
GSE64763 and GSE68312 which were downloaded from GEO (http://www.ncbi.nlm.nih.gov/geo/). In the present study, we identified DEGs between leiomyosarcoma samples and normal myometrial samples using the web tool GEO2R. Then, we performed Gene Ontology (GO) and Kyoto Encyclopaedia of Genes and Genomes (KEGG) pathway enrichment analyses for identifying enriched biological functions and pathways. In addition, we constructed protein-protein interaction (PPI) networks of the DEGs and key modules in the PPI networks to identify important genes and related pathways. Our study provides new information on the molecular aetiology and pathogenesis of uLMS and to provide novel potential molecular targets for treatment.

\section{Materials and methods}

Microarray data. After searching the GEO repository at the National Centre for Biotechnology Information (NCBI) (http://www.ncbi.nlm.nih.gov/geo/) for relevant microarray data on uLMS, the three most eligible gene expression profiles were found: GSE764, GSE64763 and GSE68312. The GSE764 profile consists of 26 samples, including 4 myometrial samples, 9 uLMS samples and some other leiomyoma and leiomyosarcoma samples. The GSE64763 profile consists of 25 fibroid samples, 25 leiomyosarcoma samples and 29 normal myometrial samples. The GSE68312 profile consists of 3 uLMS samples, 3 uterine normal myometrial samples and some samples of uterine leiomyoma tissues and cell lines of human uLMS as well as methylated forms of all these samples.

Identification of DEGs. After the irrelevant samples were excluded, the uLMS samples were compared with the normal myometrial samples using GEO2R. GEO2R, which is an interactive web tool, is designed for the identification of genes that are differentially expressed across experimental conditions by comparing two or more groups of samples in a GEO series. When analysed by the GEO2R, results were presented as a table of genes ordered by significance, then we can choose to view profile graphs of the top 250 genes or save the complete results table. In this way, the DEGs of the three gene expression profiles were identified. The genes with $\log \mathrm{FCl} \geq 1$ were regarded to be differentially expressed and $\mathrm{P}<0.05$ was considered to indicate a statistically significant difference. Then, the genes that were differentially expressed in all the three microarrays with identical expression patterns were deemed as DEGs in this study.

$G O$ analysis and KEGG pathway enrichment analysis of DEGs. The GO repository (http://geneontology.org/) consists of a large set of annotation terms and is commonly used for annotating genes and identifying the characteristic biological attributes for microarray data. The KEGG database (http://www.genome.jp/) contains data on known genes and their biochemical functions and is used for identifying functional and metabolic pathways. By performing the GO and KEGG analysis at the functional level, we can gain a better understanding of the roles of these DEGs in the initiation and in the progression of uLMS. The Database for Annotation, Visualization and Integrated Discovery (DAVID)

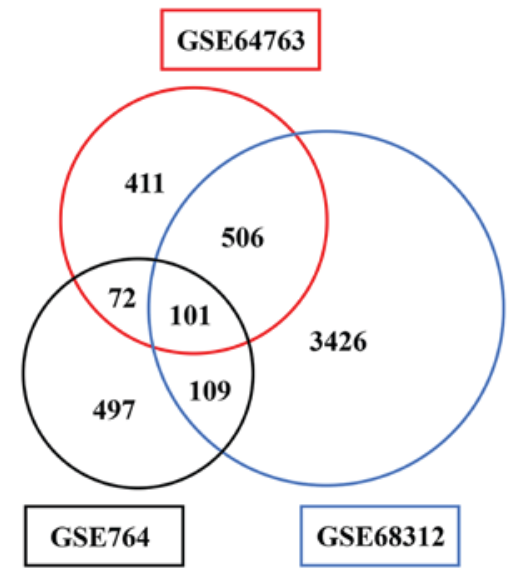

Figure 1.DEGs identified in the gene expression profiles, GSE764, GSE64763 and GSE68312.

(http://david.abcc.ncifcrf.gov/) is an online resource that provides tools for functional annotation and bioinformatics microarray analysis. Both GO categories and KEGG pathway enrichment analysis were performed using DAVID to reveal the functions of these DEGs. $\mathrm{P}<0.05$ was considered to indicate a statistically significant difference.

Protein-protein interaction (PPI) network construction and module analysis. The Search Tool for the Retrieval of Interacting Genes (STRING) database (https://string-db.org/) is a web resource of PPIs. Cytoscape (http://www.cytoscape. org/) is an open source software that allows for the visualization of molecular interaction networks and the integration of these networks with gene annotations and expression profiles. All the identified DEGs were uploaded to STRING (version 10.0) for the analysis of their interactions. Comprehensive information on the interactions of these DEGs was downloaded from STRING and the interactions with a combined score $>0.4$ were selected for constructing the PPI networks using the Cytoscape software. Next, significant modules from the PPI network were extracted using the plugin, Molecular Complex Detection (MCODE) with cut-off criteria of MCODE scores $>3$ and number of nodes $>5$. Then, the functional and pathway enrichment analyses of the genes in these modules were performed. $\mathrm{P}<0.05$ was considered to indicate a statistically significant difference.

\section{Results}

Identification of the DEGs. Analysed with GEO2R, genes which were differentially expressed between uLMS samples and normal myometrial samples were screened based on the data of GSE764, GSE64763 and GSE68312 profiles. Totally $779,1,090$ and 4,142 DEGs were identified, respectively. Among them, 101 genes were identified in all of the three datasets and 95 genes including 21 upregulated genes and 74 downregulated genes exhibited identical expression patterns (Fig. 1).

GO term and KEGG pathway enrichment analysis. The results of GO categories analysis including biological processes (BP), cellular components (CC) and molecular functions (MF) are displayed in Table I. $\mathrm{P}<0.05$ was considered as 
Table I. GO and KEGG enrichment analyses of the DEGs in uLMS.

\begin{tabular}{llrr}
\hline Category & \multicolumn{1}{c}{ Term } & Count & P-value \\
\hline Upregulated & & \\
GOTERM_BP_FAT & GO:0006259 DNA metabolic process & 5 & $6.93 \mathrm{E}-03$ \\
GOTERM_BP_FAT & GO:0034654 nucleobase-containing compound biosynthetic process & 9 & $7.24 \mathrm{E}-03$ \\
GOTERM_BP_FAT & GO:0034645 cellular macromolecule biosynthetic process & 10 & $7.48 \mathrm{E}-03$ \\
GOTERM_CC_FAT & GO:0000307 cyclin-dependent protein kinase holoenzyme complex & 2 & $3.57 \mathrm{E}-02$ \\
GOTERM_MF_FAT & GO:0001135 transcription factor activity, RNA polymerase II transcription & 2 & $8.53 \mathrm{E}-03$ \\
& factor recruiting & \\
GOTERM_MF_FAT & GO:0001134 transcription factor activity, transcription factor recruiting & $1.10 \mathrm{E}-02$ \\
GOTERM_MF_FAT & GO:0016538 cyclin-dependent protein serine/threonine kinase regulator activity & 2 & $2.66 \mathrm{E}-02$ \\
Downregulated & & \\
GOTERM_BP_FAT & GO:0070887 cellular response to chemical stimulus & 26 & $7.35 \mathrm{E}-09$ \\
GOTERM_BP_FAT & GO:0006928 movement of cell or subcellular component & 21 & $2.66 \mathrm{E}-07$ \\
GOTERM_BP_FAT & GO:0010035 response to inorganic substance & 10 & $4.83 \mathrm{E}-07$ \\
GOTERM_CC_FAT & GO:0042383 sarcolemma & 5 & $1.75 \mathrm{E}-04$ \\
GOTERM_CC_FAT & GO:0098590 plasma membrane region & 10 & $2.40 \mathrm{E}-04$ \\
GOTERM_CC_FAT & GO:0005887 integral component of plasma membrane & 13 & $1.23 \mathrm{E}-03$ \\
GOTERM_MF_FAT & GO:0005267 potassium channel activity & 5 & $1.32 \mathrm{E}-03$ \\
GOTERM_MF_FAT & GO:0015079 potassium ion transmembrane transporter activity & 5 & $2.11 \mathrm{E}-03$ \\
GOTERM_MF_FAT & GO:0015269 calcium-activated potassium channel activity & 3 & $2.41 \mathrm{E}-03$ \\
KEGG_PATHWAY & ptr05202: Transcriptional misregulation in cancer & 7 & $5.69 \mathrm{E}-04$ \\
KEGG_PATHWAY & ptr05205: Proteoglycans in cancer & 6 & $7.61 \mathrm{E}-03$ \\
KEGG_PATHWAY & ptr05200: Pathways in cancer & 7 & $3.23 \mathrm{E}-02$ \\
\hline
\end{tabular}

GO, Gene Ontology; KEGG, Kyoto Encyclopaedia of Genes and Genomes; DEGs, differentially expressed genes; uLMS, uterine leiomyosarcoma.

the cut-off value. When the number of significant terms was $>3$, only the 3 predominant terms were present. Firstly, the upregulated DEGs were annotated with the BP category, including 'DNA metabolic process', 'nucleobase-containing compound biosynthetic process' and 'cellular macromolecule biosynthetic process'; the downregulated DEGs were annotated with the GO terms, 'cellular response to chemical stimulus', 'movement of cell or subcellular component' and 'response to inorganic substance'. Secondly, the upregulated DEGs were annotated with the GO terms of the CC category, namely, 'cyclin-dependent protein kinase holoenzyme complex' and the downregulated DEGs were annotated with 'sarcolemma', 'plasma membrane region' and 'integral component of plasma membrane'. Thirdly, the upregulated DEGs were annotated with the GO terms of the MF category, such as 'transcription factor activity' ('RNA polymerase II transcription factor recruiting' and 'transcription factor recruiting') and 'cyclin-dependent protein serine/threonine kinase regulator activity'; and the downregulated DEGs were annotated with 'potassium channel activity', 'potassium ion transmembrane transporter activity' and 'calcium-activated potassium channel activity'. As shown in Table I, the significantly enriched KEGG pathways of the DEGs with $\mathrm{P}<0.05$ were 'transcriptional misregulation in cancer', 'proteoglycans in cancer' and 'pathways in cancer', all of which were pathways of downregulated DEGs. No significant pathway of upregulated DEGs was identified.
Hub genes and significant modules screened from the PPI network. After all the DEGs were uploaded to the online STRING database, the PPI network with 65 nodes and 119 edges was constructed using the Cytoscape software (Fig. 2). Sixteen hub DEGs with the node degree $>5$ were obtained (Table II). Among them, MMP9, APOE, CCNE1, $S D C 1$ and $F O X M 1$ were the major upregulated genes, while ESR1, CXCL12, AR and WT1 were the major downregulated genes. Then, one significant module that fulfilled the cut-off criteria, namely, MCODE scores $>3$ and number of nodes $>5$, was screened (Fig. 2). The SDC1, APOE, IGF1, THBS1 and CXCL12 genes were identified in the module. GO analysis of these genes showed that they were annotated with 'cell migration', 'cell motility' and 'localization of cell' (Table III). In addition, the KEGG enrichment analysis suggested that these genes were mainly involved in 'proteoglycans in cancer', 'malaria', 'p53 signalling pathway' and 'ECM-receptor interaction' (Table III). Based on the PPI network and the analysis of the significant module, the interactions between the DEGs were revealed clearly.

\section{Discussion}

uLMS is a rare tumor with unknown aetiology. All current therapies for uLMS have some limitations because of its high rates of recurrence and metastasis. Therefore, the recently emerging targeted therapy shows importance. Bioinformatics 


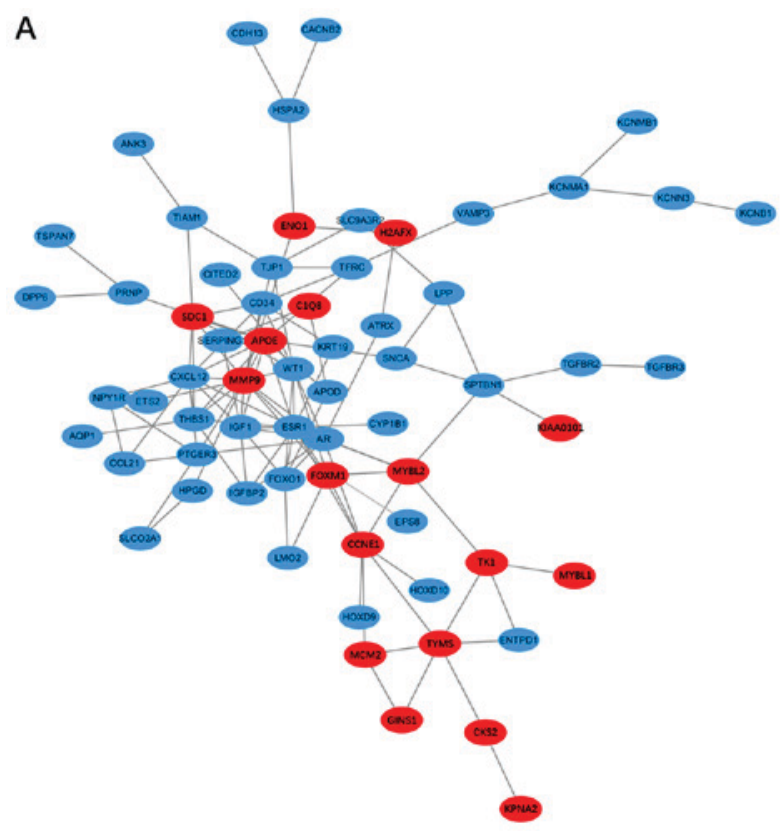

B

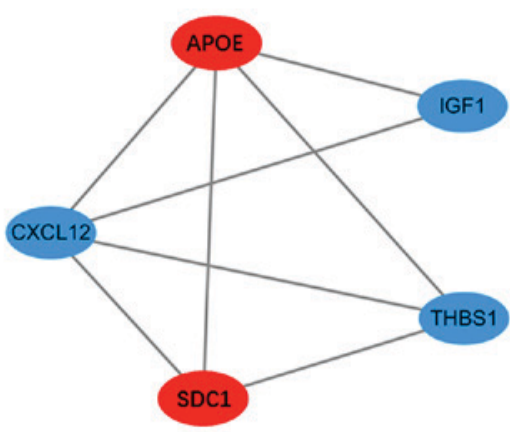

Figure 2. PPI network and the significant module. (A) PPI network of the DEGs. (B) significant module of the PPI network. Red nodes denote the upregulated genes, while blue nodes denote downregulated genes. PPI, protein-protein interaction; DEGs, differentially expressed genes.

analysis of data from high-throughput sequencing and microarrays can accurately reveal the potential molecular mechanisms of the development of uLMS and predict therapeutic targets by comparing sarcoma lesions with normal tissues. In the present study, based on the profiles, GSE764, GSE64763 and GSE68312 from the GEO repository, 21 upregulated and 74 downregulated DEGs were identified by comparing uLMS samples with normal myometrial samples. Further analysis was performed in order to explore the relations among the DEGs and the interactions of their protein products.

Functional annotation and enrichment analysis were performed using the online resource DAVID to obtain information on the biological functions of these DEGs. On the one hand, the results of the GO analysis suggested that the upregulated DEGs were annotated with 'DNA metabolic process', 'nucleobase-containing compound biosynthetic process' and 'cellular macromolecule biosynthetic process', while the downregulated DEGs were annotated with 'cellular response to chemical stimulus', 'movement of cell or subcellular component' and 'response to inorganic substance'. The increased levels of DNA replication and translation are comprehensible for the uncontrolled proliferation of cancer. On the other hand, the KEGG analysis showed that the downregulated DEGs were enriched in 'transcriptional misregulation in cancer', 'proteoglycans in cancer' and 'pathways in cancer'. As reported in the literature, transcriptional deregulation (6) as well as alteration of pathways such as the p53 signalling pathway, the Wnt signalling pathway and the PI3K/AKT/mTOR pathway were verified in many types of cancer. For example, Wnt signalling pathway was proved important in cancer progression, including tumor initiation, growth, metastasis as well as cell senescence and death in breast cancer and colonal cancer; importantly, targeting WNT signalling pathways is potential new therapy in cancer patients (7). The expression of PTEN, which is a negative regulator of PI3K/AKT/mTOR signalling pathway, was significantly reduced in more than one half of uLMS patients; what's more, the PI3K/AKT/mTOR signaling inhibitor sapanisertib and serabelisi are currently tested in clinical trials for sarcomas (8). In addition, alteration of the expression of proteoglycans during the development of cancer was also confirmed (9). Therefore, the results of the GO and KEGG analyses in our study are consistent with those of the previous studies and could provide a novel understanding of the pathogenesis of uLMS.

With the information on the interactions from STRING, the PPI network was constructed to reveal the relations among the DEGs. $M M P$ 9, also called matrix metallopeptidase 9, was the principal upregulated gene with the highest node degree. It bears direct interactions with some other key genes such as ESR 1, SDC1, APOE, THBS1, CXCL12, IGF1, FOXM1, AR and $W T 1$. As the major member of the matrix metallopeptidase family, MMP9 plays a vital role in tumor progression because of its ability to degrade the extracellular matrix. It is reported that activation of ERK drives the upregulation of MMP9 expression and subsequent MMP9 mediated shedding of SDC1 (syndecan 1) (10). According to the study by Brule et al (11), the shedding of SDC1 mediated by the MMP9 was accelerated by SDF-1/CXCL12 in HeLa cells and human primary macrophages. In addition, an MMP9-miR-494-SDC1 regulatory loop was revealed to be associated with irradiation-induced angiogenesis in medulloblastoma cells. In this regulatory mechanism, suppression of miR- 494 by MMP9 leads to the enhanced SDC1 shedding and angiogenesis (12). In addition, the overexpression of FOXM1 can upregulate the MMP9 expression by combining to its promoter, leading to the promotion of proliferation, migration and invasion of epithelial ovarian cancer cells (13). In the contrary, MMP9 expression could be inhibited by THBS1 leading to the suppression of cell invasion in colon and ovarian cancers (14). 
Table II. Hub genes of the PPI network with higher node degrees.

\begin{tabular}{llr}
\hline Hub gene & \multicolumn{1}{c}{ Gene name } & $\begin{array}{r}\text { Node } \\
\text { degree }\end{array}$ \\
\hline $\begin{array}{l}\text { Upregulated } \\
\text { MMP9 }\end{array}$ & Matrix metallopeptidase 9 & 15 \\
APOE & Apolipoprotein E & 10 \\
CCNE1 & Cyclin E1 & 8 \\
SDC1 & Syndecan 1 & 7 \\
FOXM1 & Forkhead box M1 & 7 \\
TYMS & Thymidylate synthetase & 6 \\
MYBL2 & MYB proto-oncogene like 2 & 6 \\
Downregulated & & \\
ESR1 & Estrogen receptor 1 & 14 \\
CXCL12 & C-X-C motif chemokine ligand 12 & 10 \\
AR & Androgen receptor & 10 \\
WT & Wilms tumor 1 & 9 \\
IGF1 & Insulin like growth factor 1 & 8 \\
THBS1 & Thrombospondin 1 & 7 \\
FOXO1 & Forkhead box O1 & 6 \\
PTGER3 & Prostaglandin E receptor 3 & 6 \\
CD34 & Cluster of differentiation & 6 \\
& 34 molecule & \\
\hline
\end{tabular}

PPI, protein-protein interaction.

Furthermore, MMP9 was also reported to activate latent cytokines and growth factors (15). In breast carcinoma, it was demonstrated that fibroblasts could promote angiogenesis and then, enhance tumor growth by the upregulation of $M M P 9$ via the MAPK-AP1 signalling axis, which is co-stimulated by TGF- $\beta$, TNF- $\alpha$ and IL- $\beta$ (15). In colorectal cancer (CRC), MMP9 was overexpressed in each clinical stage and could be used as a diagnostic marker (16). More notably, the significant negative correlation between the inhibition of Matrigel invasion and MMP9 levels in SK-UT-1 uLMS cells found by Roomi et al (17) was in support of our study. Contrary to MMP9, ESR1, which encodes oestrogen receptor 1 (ER1), was the principal downregulated gene with the most connections with other genes. In consonance with our results, the loss of ER1 activity was reported in ULMS relative to ULMY $(18,19)$. Moreover, the expression of ER1 was statistically related to survival in patients with uLMS and IHC testing of ER1 in these patients was recommended (20). However, as reported by Garcia et al (21), the expression of ER1 was not significantly correlated with the survival in patients with uLMS. According to the literature, ER1 was expressed in $40-80 \%$ of patients with uLMS and longer progression-free-survival (PFS) was observed in patients with advanced uLMS with strongly expressed ER and progesterone receptor (PR) when treated with aromatase inhibitors (22). Therefore, MMP9 and ESR 1 may play crucial roles in the progression of uLMS and may be of great value as prognosis markers.

Further, module analysis of the PPI network and the enrichment analyses were performed. Results showed that the main module was primarily involved in 'cell migration' and 'cell motility', while the enrichment pathways were 'proteoglycans in cancer', 'malaria', 'p53 signalling pathway' and 'ECM-receptor interaction'. The upregulated SDC1 and APOE genes and the downregulated THBS1, $C X C L 12$ and IGF1 genes are all hub genes of the significant module. Among these genes, only the expression of THBSI (thrombospondin 1) has been studied in uLMS and the results of most studies were consistent with our results. For instance, THBS1 was identified as a gene encoded by a BAC clone, whose expression was frequently lost in uLMS (23); THBS1 expression was moderate in ULMY but minimal in uLMS (24); THBS1 was less frequently expressed in uLMS than in $\mathrm{uLMY}$ with a significantly negative correlation between its expression and lymph-vascular space invasion in uLMS (25). THBS1, which is a secreted protein, functions as an endogenous anti-angiogenic agent with the ability to interfere with endothelial cell migration and survival. It was reported that the loss of THBS1 expression was related to worsening of PFS due to the abrogation of the suppressive effects on angiogenesis and metastasis (14). The suppression of THBS1 by the activation of the $\beta$-adrenergic signalling pathway could induce angiogenesis in prostate cancer (26). What's more, research indicated that SDC1 could be necessary in coupling between THBS1 and fascin spike formation, resulting in the promotion of cell spreading and cytoskeletal organization (27). SDC1 encodes the type1 transmembrane heparan sulphate proteoglycan (HSPG) that contributes substantially to the cell-cell and cell-matrix interactions, cell growth and migration, neovascularisation and adhesion-dependent signalling pathways. As reported by Alexander et al (28), SDC1 is essential for tumorigenesis, which is induced by the Wnt-1 signalling pathway in mouse mammary gland. By interacting with laminin 332, SDC1 could promote tumor invasion via the PI $3 \mathrm{~K}$ and RAC1 signalling pathways (29). Clinically, SDCl has proved to be valuable as a prognostic marker in metastatic CRC patients (30). In addition, SDC1 was found to internalize Apolipoprotein E-very low-density lipoproteins (apoE-VLDL) in human fibroblasts through a low density lipoprotein receptor-related protein (LRP)-independent pathway (31). ApoE, encoded by gene $A P O E$, is mainly produced by the liver and the macrophages of peripheral tissues and astrocytes of the brain. As a major component of low-density lipoproteins (LDL) and VLDL, ApoE mediates the metabolism and transport of lipoproteins (32). It is noteworthy that ApoE is related to tumorigenesis. For example, serum ApoE levels were strikingly elevated in non-small cell lung cancer (NSCLC) patients (33) as well as in breast cancer patients (34) relative to normal healthy controls; meanwhile, the elevated levels of ApoE were correlated with tumor metastasis and poor prognosis. Furthermore, in gastric cancer, the DEGs related to ApoE such as the upregulated transcription factors, signal transducer and activator of transcription 2 (STAT2) and STAT3 were mainly involved in the JAK-STAT cascade and the steroid hormone response (35). However, different from current views, $C X C L 12$ and $I G F 1$, which are usually related to tumor invasion and metastasis, are downregulated in uLMS which is a finding of our study. CXCL12, which is also known as stromal cell-derived factor 1 (SDF1) or pre-B 
Table III. GO and KEGG enrichment analyses of genes in the significant module of the PPI network.

\begin{tabular}{|c|c|c|c|c|}
\hline Category & Term & Count & P-value & Genes \\
\hline GOTERM_BP_FAT & GO:0016477 cell migration & 5 & $1.23 \mathrm{E}-05$ & $\begin{array}{l}\text { SDC1, APOE, IGF1, THBS1, } \\
\text { CXCL12 }\end{array}$ \\
\hline GOTERM_BP_FAT & GO:0048870 cell motility & 5 & $1.93 \mathrm{E}-05$ & $\begin{array}{l}\text { SDC1, APOE, IGF1, THBS1, } \\
\text { CXCL12 }\end{array}$ \\
\hline GOTERM_BP_FAT & GO:0051674 localization of cell & 5 & $1.93 \mathrm{E}-05$ & $\begin{array}{l}\text { SDC1, APOE, IGF1, THBS1, } \\
\text { CXCL12 }\end{array}$ \\
\hline GOTERM_CC_FAT & $\begin{array}{l}\text { GO:0009897 external side of } \\
\text { plasma membrane }\end{array}$ & 3 & $1.23 \mathrm{E}-03$ & SDC1, THBS1, CXCL12 \\
\hline GOTERM_CC_FAT & GO:0031988 membrane-bounded vesicle & 5 & $2.18 \mathrm{E}-03$ & $\begin{array}{l}\text { SDC1, APOE, IGF1, THBS1, } \\
\text { CXCL12 }\end{array}$ \\
\hline GOTERM_CC_FAT & GO:0098552 side of membrane & 3 & $3.63 \mathrm{E}-03$ & SDC1, THBS1, CXCL12 \\
\hline GOTERM_MF_FAT & GO:0071813 lipoprotein particle binding & 2 & 4.77E-03 & APOE, THBS1 \\
\hline GOTERM_MF_FAT & GO:0071814 protein-lipid complex binding & 2 & 4.77E-03 & APOE, THBS1 \\
\hline GOTERM_MF_FAT & GO:0005102 receptor binding & 3 & $1.05 \mathrm{E}-02$ & APOE, IGF1, CXCL12 \\
\hline KEGG_PATHWAY & ssc05205: Proteoglycans in cancer & 3 & $4.21 \mathrm{E}-03$ & SDC1, IGF1, THBS1 \\
\hline KEGG_PATHWAY & ssc05144: Malaria & 2 & $2.93 \mathrm{E}-02$ & SDC1, THBS1 \\
\hline KEGG_PATHWAY & ssc04115: p53 signaling pathway & 2 & 4.04E-02 & IGF1, THBS1 \\
\hline KEGG_PATHWAY & ssc04512: ECM-receptor interaction & 2 & 4.53E-02 & SDC1, THBS1 \\
\hline
\end{tabular}

GO, Gene Ontology; KEGG, Kyoto Encyclopaedia of Genes and Genomes; PPI, protein-protein interaction.

cell stimulating factor (PBSF), is a chemokine that mediates inflammatory response, regulates stem cell migration and participates in tumor metastasis. CXCL12 regulates multiple tumor-related factors via the CXCL12-CXCR4 axis and finally results in tumor progression in various cancers. However, Roy et al (36) found that the expression of CXCL12 was low in pancreatic cancer tissue relative to healthy tissue and they concluded that the expression of CXCL12 suppressed tumor growth and metastasis in pancreatic cancer based on the results of their study. Besides, CXCL12 was identified as the target of oestrogen in ER-positive ovarian cancer and breast cancer and ER-positive cell lines could express CXCL12 with the existence of oestradiol (37). As mentioned previously, the expression of ER was low in uLMS. Collectively, it is possible that $C X C L 12$ is downregulated in uLMS; however, further studies are required for understanding its effect. Insulin-like growth factor 1 (IGF1) is an important polypeptide growth factor which could promote proliferation and inhibit apoptosis by activating the PI3K-Akt and MAPK pathways in cancer. At present, there is no report on the expression or effect of IGF1 in uLMS. The major significant genes and pathways were identified based on the results of the module analysis and the enrichment analysis, which may help in the better understanding of the mechanism of the development of uLMS.

In addition to the abovementioned genes, CCNE1, FOXM1, $A R$ and $W T 1$ are also crucial hub genes. CCNE1 and FOXM1, both trigger cancer; however, no study on their role in uLMS has been reported. Cyclin E1 (CCNE1), a key regulator of the G1/S transition, suppresses the retinoblastoma (RB) protein by activating cyclin-dependent kinases (CDK), leading to unrestricted proliferation. It was reported that $C C N E 1$ was overexpressed in breast cancer (38) as well as in ovarian cancer (39) and could be used as a potential target for ovarian cancer therapy $(39,40)$. FOXM1, a transcriptional factor with the fork head domain, is related to proliferation, angiogenesis metastasis, which are vital events of tumorigenesis and tumor progression $(41,42)$. FOXM1 was identified as a driver of tumor progression and potential clinical marker in aggressive cancers, such as prostate cancer and breast cancer and the suppression of FOXM1 was regarded as a promising method of cancer therapy $(41,42)$. In contrast to CCNEI and FOXM1, $A R$ and $W T 1$ were the downregulated genes identified in our analyses. $A R$ is the gene that encodes the androgen receptor. In the study by Koivisto-Korander et al (20), AR immunoreactivity was absent in all 100 uterine sarcoma samples including 28 uLMS samples. Similarly, AR was found to be related to a lower risk of recurrence of uLMS by Leitao et al (43). Wilms tumor-1 (WT1) protein, is a transcription factor as well as a tumor suppressor. Mutations in $W T 1$ were reported to relate to childhood tumors of the kidney (44). Studies suggested that $W T 1$ was less expressed in uLMS than in uLMY (45) and patients with WT1-negative high-grade uterine sarcoma had a better prognosis than the patients with WT1-positive tumors (46). From the abovementioned findings, we conclude that AR and WT1 could be used as prognostic markers, while CCNE1 and FOXM1 may also participate in the development of uLMS and more studies are required to reveal the underlying mechanisms.

In conclusion, a total of 95 DEGs including 21 upregulated genes and 74 downregulated genes were identified in the uLMS samples. As per the PPI network and module analysis, the DEGs are enriched in 'proteoglycans in cancer', 'p53 signalling pathway' and 'ECM-receptor interaction'. In addition, $M M P 9$, $A P O E, S D C 1, C C N E 1$ and $F O X M 1$ may play predominant roles 
in the initiation and progression of uLMS. This study is the first to identify the key DEGs and related pathways as well as the interactions among these key genes in uLMS using bioinformatic analysis, which may provide a novel understanding of the underlying mechanisms and help in discovering new molecular targets for the treatment of uLMS. However, there are limitations of the present study, including relatively small sample size and no targeted experimental validation. Therefore, further studies are needed in the future.

\section{Acknowledgements}

Not applicable.

\section{Funding}

The present study was supported by the Natural Science Foundation of China (grant nos. 81572568 and 81272863).

\section{Availability of data and materials}

The datasets analyzed during the current study are available in the GEO repository (http://www.ncbi.nlm.nih.gov/geo/).

\section{Authors' contributions}

YZa, YW and FX conceived and designed the study. YZa, LG and YZh performed the study. YZa wrote the paper. YW and FX revised and edited the manuscript. All authors read and approved the final manuscript.

\section{Ethics approval and consent to participate}

Not applicable.

\section{Consent for publication}

Not applicable.

\section{Competing interests}

The authors declare that they have no competing interests.

\section{References}

1. Ricci S, Stone RL and Fader AN: Uterine leiomyosarcoma: Epidemiology, contemporary treatment strategies and the impact of uterine morcellation. Gynecol Oncol 145: 208-216, 2017.

2. Park JY, Lee JW, Lee HJ, Lee JJ, Moon SH, Kang SY, Cheon GJ and Chung HH: Prognostic significance of preoperative ${ }^{18} \mathrm{~F}-\mathrm{FDG}$ PET/CT in uterine leiomyosarcoma. J Gynecol Oncol 28: e28, 2017.

3. Ducie JA and Leitao MM Jr: The role of adjuvant therapy in uterine leiomyosarcoma. Expert Rev Anticancer Ther 16: 45-55, 2016.

4. Cui RR, Wright JD and Hou JY: Uterine leiomyosarcoma: A review of recent advances in molecular biology, clinical management and outcome. BJOG 124: 1028-1037, 2017.

5. Barlin JN, Zhou QC, Leitao MM, Bisogna M, Olvera N, Shih KK, Jacobsen A, Schultz N, Tap WD, Hensley ML, et al: Molecular subtypes of uterine leiomyosarcoma and correlation with clinical outcome. Neoplasia 17: 183-189, 2015

6. Lee TI and Young RA: Transcriptional regulation and its misregulation in disease. Cell 152: 1237-1251, 2013.

7. Anastas JN and Moon RT: WNT signalling pathways as therapeutic targets in cancer. Nat Rev Cancer 13: 11-26, 2013.
8. Cuppens T, Moisse M, Depreeuw J, Annibali D, Colas E, Gil-Moreno A, Huvila J, Carpén O,Zikán M, Matias-Guiu X, et al: Integrated genome analysis of uterine leiomyosarcoma to identify novel driver genes and targetable pathways. Int J Cancer 142: 1230-1243, 2018 .

9. Iozzo RV and Sanderson RD: Proteoglycans in cancer biology, tumor microenvironment and angiogenesis. J Cell Mol Med 15: 1013-1031, 2011

10. Purushothaman A, Babitz SK and Sanderson RD: Heparanase enhances the insulin receptor signaling pathway to activate extracellular signal-regulated kinase in multiple myeloma. J Biol Chem 287: 41288-41296, 2012.

11. Brule S, Charnaux N, Sutton A, Ledoux D, Chaigneau T, Saffar L and Gattegno L: The shedding of syndecan-4 and syndecan-1 from HeLa cells and human primary macrophages is accelerated by SDF-1/CXCL12 and mediated by the matrix metalloproteinase-9. Glycobiology 16: 488-501, 2006.

12. Asuthkar S, Velpula KK, Nalla AK, Gogineni VR, Gondi CS and Rao JS: Irradiation-induced angiogenesis is associated with an MMP-9-miR-494-syndecan-1 regulatory loop in medulloblastoma cells. Oncogene 33: 1922-1933, 2014.

13. Wen N, Wang Y, Wen L, Zhao SH, Ai ZH, Wang Y, Wu B, Lu HX, Yang H, Liu WC and Li Y: Overexpression of FOXM1 predicts poor prognosis and promotes cancer cell proliferation, migration and invasion in epithelial ovarian cancer. J Transl Med 12: 134, 2014.

14. Tzeng HT, Tsai CH, Yen YT, Cheng HC, Chen YC, Pu SW, Wang YS, Shan YS, Tseng YL, Su WC, et al: Dysregulation of Rab37-mediated cross-talk between cancer cells and endothelial cells via thrombospondin-1 promotes tumor neovasculature and metastasis. Clin Cancer Res 23: 2335-2345, 2017.

15. Limoge M, Safina A, Beattie A, Kapus L, Truskinovsky AM and Bakin AV: Tumor-fibroblast interactions stimulate tumor vascularization by enhancing cytokine-driven production of MMP9 by tumor cells. Oncotarget 8: 35592-35608, 2017.

16. Lorenc Z, Waniczek D, Lorenc-Podgórska K, Krawczyk W, Domagała M, Majewski M and Mazurek U: Profile of expression of genes encoding matrix metallopeptidase 9 (MMP9), matrix metallopeptidase 28 (MMP28) and TIMP metallopeptidase inhibitor 1 (TIMP1) in colorectal cancer: Assessment of the role in diagnosis and prognostication. Med Sci Monit 23: 1305-1311, 2017.

17. Roomi MW, Kalinovsky T, Niedzwiecki A and Rath M: Modulation of u-PA, MMPs and their inhibitors by a novel nutrient mixture in adult human sarcoma cell lines. Int $\mathbf{J}$ Oncol 43: 39-49, 2013.

18. Lusby K, Savannah KB, Demicco EG, Zhang Y, Ghadimi MP, Young ED, Colombo C, Lam R, Dogan TE, Hornick JL, et al: Uterine leiomyosarcoma management, outcome, and associated molecular biomarkers: A single institution's experience. Ann Surg Oncol 20: 2364-2372, 2013.

19. Zhu XQ, Shi YF, Cheng XD and Wu YZ: The differential diagnosis between uterine leiomyosarcoma and the special subtypes of leiomyoma. Zhonghua Yi Xue Za Zhi 83: 1419-1421, 2003 (In Chinese).

20. Koivisto-Korander R, Butzow R, Koivisto AM and Leminen A: Immunohistochemical studies on uterine carcinosarcoma, leiomyosarcoma, and endometrial stromal sarcoma: Expression and prognostic importance of ten different markers. Tumour Biol 32: 451-459, 2011.

21. Garcia C, Kubat JS, Fulton RS, Anthony AT, Combs M, Powell CB and Littell RD: Clinical outcomes and prognostic markers in uterine leiomyosarcoma: A population-based cohort. Int J Gynecol Cancer 25: 622-628, 2015.

22. George S, Feng Y, Manola J, Nucci MR, Butrynski JE, Morgan JA, Ramaiya N, Quek R, Penson RT, Wagner AJ, et al: Phase 2 trial of aromatase inhibition with letrozole in patients with uterine leiomyosarcomas expressing estrogen and/or progesterone receptors. Cancer 120: 738-743, 2014.

23. Cho YL, Bae S, Koo MS, Kim KM, Chun HJ, Kim CK, Ro DY, Kim JH, Lee CH, Kim YW and Ahn WS: Array comparative genomic hybridization analysis of uterine leiomyosarcoma. Gynecol Oncol 99: 545-551, 2005.

24. Uluer ET, Inan S, Ozbilgin K, Karaca F, Dicle N and Sanci M: The role of hypoxia related angiogenesis in uterine smooth muscle tumors. Biotech Histochem 90: 102-110, 2015.

25. Bodner-Adler B, Nather A, Bodner K, Czerwenka K, Kimberger O, Leodolter S and Mayerhofer K: Expression of thrombospondin 1 (TSP 1) in patients with uterine smooth muscle tumors: An immunohistochemical study. Gynecol Oncol 103: 186-189, 2006. 
26. Hulsurkar M, Li Z, Zhang Y, Li X, Zheng D and Li W: Beta-adrenergic signaling promotes tumor angiogenesis and prostate cancer progression through HDAC2-mediated suppression of thrombospondin-1. Oncogene 36: 1525-1536, 2017.

27. Adams JC, Kureishy N and Taylor AL: A role for syndecan-1 in coupling fascin spike formation by thrombospondin-1. J Cell Biol 152: 1169-1182, 2001.

28. Alexander CM, Reichsman F, Hinkes MT, Lincecum J, Becker KA, Cumberledge S and Bernfield M: Syndecan-1 is required for Wnt-1-induced mammary tumorigenesis in mice. Nat Genet 25: 329-332, 2000.

29. Marinkovich MP: Tumor microenvironment: Laminin 332 in squamous-cell carcinoma. Nat Rev Cancer 7: 370-380, 2007.

30. Jary M, Lecomte T, Bouché O, Kim S, Dobi E, Queiroz L, Ghiringhelli F, Etienne H, Léger J, Godet Y, et al: Prognostic value of baseline seric Syndecan-1 in initially unresectable metastatic colorectal cancer patients: A simple biological score. Int J Cancer 139: 2325-2335, 2016.

31. Wilsie LC, Gonzales AM and Orlando RA: Syndecan-1 mediates internalization of apoE-VLDL through a low density lipoprotein receptor-related protein (LRP)-independent, non-clathrin-mediated pathway. Lipids Health Dis 5: 23, 2006.

32. Huang YA, Zhou B, Wernig M and Südhof TC: ApoE2, ApoE3, and ApoE4 differentially stimulate APP transcription and $\mathrm{A} \beta$ secretion. Cell 168: 427-441.e21, 2017.

33. Luo J, Song J, Feng P, Wang Y, Long W, Liu M and Li L: Elevated serum apolipoprotein $\mathrm{E}$ is associated with metastasis and poor prognosis of non-small cell lung cancer. Tumour Biol 37: 10715-10721, 2016.

34. Xu X, Wan J, Yuan L, Ba J, Feng P, Long W, Huang H, Liu P, Cai Y, Liu M, et al: Serum levels of apolipoprotein E correlates with disease progression and poor prognosis in breast cancer. Tumour Biol: Oct 5, 2016 (Epub ahead of print).

35. Shi X, Xu J, Wang J, Cui M, Gao Y, Niu H and Jin H: Expression analysis of apolipoprotein $\mathrm{E}$ and its associated genes in gastric cancer. Oncol Lett 10: 1309-1314, 2015.

36. Roy I, Zimmerman NP, Mackinnon AC, Tsai S, Evans DB and Dwinell MB: CXCL12 chemokine expression suppresses human pancreatic cancer growth and metastasis. PLoS One 9: e90400, 2014.

37. Jiang YP and Wu XH: Correlations of chemokine CXCL12 and its receptor to tumor metastasis. Ai Zheng 26: 220-224, 2007 (In Chinese)
38. Wu Y, Guo X, Brandt Y, Hathaway HJ and Hartley RS Three-dimensional collagen represses cyclin E1 via $\beta 1$ integrin in invasive breast cancer cells. Breast Cancer Res Treat 127: 397-406, 2011

39. Nakayama N, Nakayama K, Shamima Y, Ishikawa M, Katagiri A, Iida K and Miyazaki K: Gene amplification CCNE1 is related to poor survival and potential therapeutic target in ovarian cancer. Cancer 116: 2621-2634, 2010.

40. Kanska J, Zakhour M, Taylor-Harding B, Karlan BY and Wiedemeyer WR: Cyclin E as a potential therapeutic target in high grade serous ovarian cancer. Gynecol Oncol 143: 152-158, 2016.

41. Lin SC, Kao CY, Lee HJ, Creighton CJ, Ittmann MM, Tsai SJ, Tsai SY and Tsai MJ: Dysregulation of miRNAs-COUP-TFII-FOXM1-CENPF axis contributes to the metastasis of prostate cancer. Nat Commun 7: 11418, 2016.

42. Gormally MV, Dexheimer TS, Marsico G, Sanders DA, Lowe C, Matak-Vinković D, Michael S, Jadhav A, Rai G, Maloney DJ, et al: Suppression of the FOXM1 transcriptional programme via novel small molecule inhibition. Nat Commun 5: $5165,2014$.

43. Leitao MM, Soslow RA, Nonaka D, Olshen AB, Aghajanian C, Sabbatini P, Dupont J, Hensley M, Sonoda Y, Barakat RR and Anderson S: Tissue microarray immunohistochemical expression of estrogen, progesterone, and androgen receptors in uterine leiomyomata and leiomyosarcoma. Cancer 101: 1455-1462, 2004.

44. Nachtigal MW, Hirokawa Y, Enyeart-VanHouten DL, Flanagan JN, Hammer GD and Ingraham HA: Wilms' tumor 1 and Dax-1 modulate the orphan nuclear receptor SF-1 in sex-specific gene expression. Cell 93: 445-454, 1998.

45. Patil DT, Laskin WB, Fetsch JF and Miettinen M: Inguinal smooth muscle tumors in women-a dichotomous group consisting of Müllerian-type leiomyomas and soft tissue leiomyosarcomas: An analysis of 55 cases. Am J Surg Pathol 35: 315-324, 2011.

46. Coosemans A, Van Calster B, Verbist G, Moerman P, Vergote I, Van Gool SW and Amant F: Wilms tumor gene 1 (WT1) is a prognostic marker in high-grade uterine sarcoma. Int J Gynecol Cancer 21: 302-308, 2011 